\title{
Alimentation de la jument lourde allaitante : évolution du poids vif des juments et croissance des poulains
}

\author{
W. MARTIN-ROSSET, M. DOREAU *, J. ESPINASSE \\ avec la collaboration de $\mathbf{H}$. DUbroeucQ et $\mathbf{R}$. JAILleR** \\ Unité Elevage et Alimentation du Cheval \\ * Laboratoire de la Lactation \\ **: Laboratoire de la Production de Viande \\ INRA, C.R.Z.V. de Theix, F 63122 Ceyrat
}

\begin{abstract}
Résumé
L'évolution du poids vif de la jument lourde allaitante bien alimentée au cours du cycle annuel de gestation-lactation, et de la croissance du poulain allaité a été étudiée.

Au cours des 3 derniers mois de gestation le poids vif de la jument s'accroît de 8 p. 100 . Ce gain de poids correspond à l'augmentation du poids du conceptus, fœtus essentiellement, et représente 73 p. 100 du poids du fretus à la naissance. Le poids de la jument s'accroît de 6 à 7 p. 100 au cours du mois suivant la mise bas. La variation de poids du contenu digestif pourrait expliquer en partie du moins le fléchissement du poids vif de la jument au cours des derniers jours de gestation comme son augmentation en début de lactation. On n'observe pas de différence selon la date de mise bas par rapport à la mise à l'herbe.

Le poulain a un poids vif à la naissance qui représente $10 \mathrm{p} .100$ du poids vif de la mère, et est lié à son âge. La croissance de la naissance au sevrage est en moyenne de $1300 \mathrm{~g} / \mathrm{j}$ : elle est très élevée au cours du premier mois où le poulain double son poids de naissance car elle est très liée à la production laitière de la mère. Il n'y a pas de différence significative selon le sexe du jeune et la date de naissance par rapport à la mise à l'herbe.

Le poids vif de la jument est un critère utile mais encore insuffisant pour apprécier l'effet du niveau des apports alimentaires au cours du cycle gestation-lactation.

La croissance du poulain est un bon critère pour estimer la production laitière de la jument et ses variations avec le niveau des apports alimentaires au cours du premier mois de lactation.
\end{abstract}

Mots clés : cheval, alimentation, jument, poulain, poids vif.

\section{Introduction}

L'alimentation des troupeaux de juments lourdes destinées à produire des poulains de boucherie a été peu étudiée, excepté en U.R.S.S. où les juments sont exploitées 
pour produire du lait et de la viande (ANASHINA \& Koveshnikov, 1982 ; Barmintsev, 1982 ; IsAEva, 1982). En effet, l'orientation de nos anciennes races de trait vers la boucherie est encore récente, à peine 15 années, et se développe surtout dans les zones de montagne, et à un moindre degré, herbagères de plaine, à vocation extensive où des surfaces peu productives sont encore sous-exploitées ou abandonnées (RossiER, 1984).

La jument lourde est le plus souvent conduite en plein air intégral, souvent en complément d'un troupeau de vaches (Martin-Rosset \& Trillaud-GeyL, 1984). Elle met bas en moyenne au début avril en région de montagne, un poulain qu'elle allaite pendant le dernier mois d'hiver puis au pâturage; ou au début mai en région de plaine, un poulain qu'elle allaite presque exclusivement au pâturage. Le poulain est sevré à l'âge de 6-7 mois, au plus tard vers la mi-octobre. La jument commence alors une longue période de restriction alimentaire qui se déroule du $4^{\circ}$ au dernier mois de gestation pour les juments conduites en région de plaine et $\mathrm{du} 5^{\mathrm{e}}$ mois de gestation au $1^{\text {"r }}$ mois de lactation pour les juments exploitées en région de montagne. La jument connaît après la mise à l'herbe une période d'alimentation plus libérale, quantitativement au moins, et qui correspond au moment où la jument peut et doit être fécondée. A cette époque elle pâture des prairies médiocres et non fauchables puis monte tôt en altitude, dans les régions de montagne, pour exploiter pendant tout l'été des pâturages peu productifs (Martin-Rosset, Loiseau \& Molenat, 1981), ou pâture en régions de plaine des refus laissés par les bovins ou exploite l'herbe en association avec ceux-ci.

Il est donc nécessaire de déterminer, chez la jument, les apports alimentaires hivernaux minima, compatibles avec une bonne santé de la poulinière et de son poulain. La jument doit en effet être fécondée puis mettre bas un poulain à la bonne saison, à un intervalle régulier de 12 mois, et avoir une production laitière suffisante pour assurer un gain de poids correct du poulain qui doit doubler son poids de naissance à l'âge d'un mois (ButAye, 1966 ; MARTIN-Rosset, 1983) et atteindre au sevrage un poids vif de $350 \mathrm{~kg}$.

Le niveau minimum des apports alimentaires aux différentes phases du cycle gestation-lactation est encore mal connu chez la jument. Tous les travaux réalisés s'intéressent exclusivement à la phase critique de la fin de la gestation et du début de la lactation (revue de Martin-Rosset \& Doreau, 1984). Ils concernent essentiellement la jument de selle conduite en box ou en semi-plein air et recevant des rations très concentrées.

L'évolution du poids vif de la jument qui traduit en particulier les variations des réserves corporelles (Sutton, Bowland \& Ratcliff, 1977; Martin-Rosset \& Doreau, 1980 ; Henneke, Potter \& Kreider, 1981 ; Goater et al., 1981 ; ZimmerMAN, 1981 ; PAGAN \& HINTZ, 1981), et la croissance du poulain qui est directement liée à la production laitière de la mère, au moins au cours du $1^{\text {er }}$ mois de lactation (Doreau, Martin-Rosset \& Dubroeuco, 1982 ; Doreau et al., 1986) paraissent des critères utiles pour apprécier l'influence du niveau des apports alimentaires au cours du cycle gestation-lactation. L'étude entreprise a pour objectifs de décrire au cours du cycle annuel de gestation-lactation chez la jument lourde bien alimentée, l'évolution du poids vif de la jument et de la croissance du poulain afin de pouvoir ultérieurement mesurer l'effet de la variation du niveau et de la nature des apports alimentaires par rapport à une couverture stricte des besoins sur les performances de la jument et de son poulain. 


\section{Matériel et méthodes}

\section{A. Mode de conduite des troupeaux}

Les juments lourdes étudiées $(\mathrm{n}=35)$ sont pour la plupart de races Comtoise ou Bretonne ou croisées. Elles ont été conduites en 2 troupeaux devant pouliner avant mise à l'herbe $(n=23)$ ou après $(n=12)$. Elles vivent en plein air intégral entre 800 et $1400 \mathrm{~m}$ d'altitude, toute l'année, en particulier pendant la période hivernale qui couvre la fin de la gestation $\left(8^{\circ}-11^{*}\right.$ mois) et le début de la lactation (1 $1^{\mathrm{er}}$ mois).

Les juments sont saillies par deux étalons munis d'un harnais marqueur (ARCHAMBEAUD et al., 1973) et effectuant la monte en liberté du $1^{\text {er }}$ février au 30 juin. Les étalons sont introduits dans les troupeaux avant les premières mises-bas qui ont eu lieu en plein air, en moyenne le 16 avril ( \pm 20 jours).

Les poulinières hivernent dans des pares à $800 \mathrm{~m}$ d'altitude de la mi-janvier à fin avril (fig. 1). Elles reçoivent alors un régime à base de foin de pré distribué en quantité limitée. En complément, $2 \mathrm{~kg}$ d'aliment concentré sont distribués par jour et individuellement aux juments en fin de gestation et en début de lactation, grâce à un système à logettes (tabl. 1 et 2).

Mode de conduite des animaux - Type of management

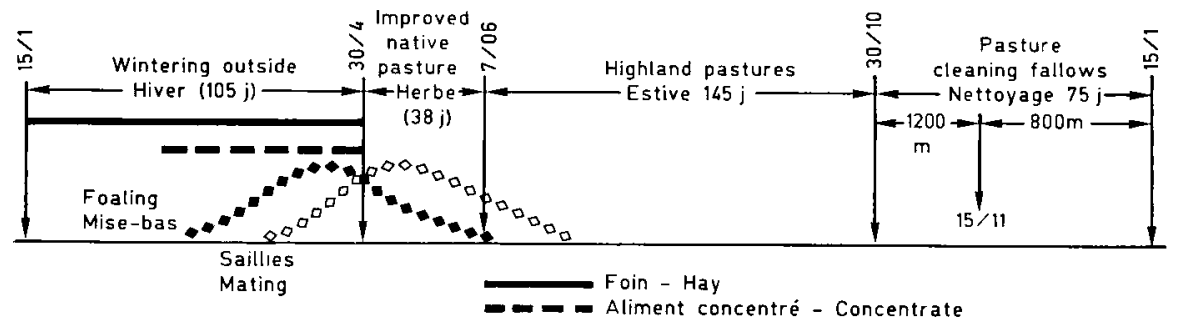

FIG. 1

Mode de conduite des animaux.

Type of management.

Les troupeaux sont mis à l'herbe fin avril à $800 \mathrm{~m}$ d'altitude (fig. 1). Ils effectuent soit un déprimage des prairies permanentes de fauche, à base de graminées fourragères 38 p. 100 (Poa communis et pratensis, Dactylis glomerata) et de légumineuses 13 p. 100 (Trifolium repens), soit le plus souvent un premier cycle de pâturage sur des prairies naturelles non fauchables ou de qualité moyenne. A la mi-juin, les poulinières montent en estive à $1400 \mathrm{~m}$ dans le Massif des Monts-Dore, pour exploiter pendant 120 jours environ des prairies d'altitude entretenues à base d'espèces fourragères 54 p. 100 dont 40 p. 100 de graminées fourragères : bonnes : Poa pratensis, Phleum pratense, ou moyennes : Agrostis tenuis et Festuca rubra (Groupe DE Recherches 
Sur les hauts-Paturages dégradés des Monts-Dore, 1979) (fig. 1). A partir de la mi-octobre (fig. 1) jusqu'à la mi-janvier, elles exploitent les refus laissés par les bovins dans les prés situés à $1200 \mathrm{~m}$ puis à $800 \mathrm{~m}$ d'altitude.

\section{Tableau 1}

Composition chimique et valeur nutritive.

Chemical composition and nutritive value of the feedstuffs.

\begin{tabular}{|c|c|c|}
\hline $\begin{array}{l}\text { Aliments } \\
\text { Feedstuffs }\end{array}$ & $\begin{array}{l}\text { Foin } \\
\text { Hay }\end{array}$ & $\begin{array}{l}\text { Aliment } \\
\text { concentré } \\
\text { Concentrate }\end{array}$ \\
\hline $\begin{array}{l}\text { Composition chimique (en p. } 100 \text { de la MS) } \ldots \\
\text { Chemical composition (\% DM) }\end{array}$ & & \\
\hline $\begin{array}{l}\text { Matières minérales } \ldots \ldots \ldots \ldots \ldots \ldots \ldots \ldots \\
\text { Ash }\end{array}$ & 6,7 & 10,7 \\
\hline $\begin{array}{l}\text { Cellulose brute } \ldots \ldots \ldots \ldots \ldots \ldots \ldots \ldots \ldots \ldots \ldots \ldots \\
\text { Crude fiber }\end{array}$ & 33,4 & 7,0 \\
\hline $\begin{array}{l}\text { Matières azotées totales } \ldots \ldots \ldots \ldots \ldots \ldots \ldots \ldots \ldots \\
\text { Crude protein }\end{array}$ & 9,4 & 13,9 \\
\hline $\begin{array}{l}\text { Valeur nutritive } \\
\text { Nutritive value }\end{array}$ & & \\
\hline $\begin{array}{l}\text { Energie : UFC }\left(/ \mathrm{kg} \text { MS) }{ }^{(1)} \ldots \ldots \ldots \ldots \ldots \ldots\right. \\
\text { Energy : } H F U\left(\begin{array}{ll}(/ \mathrm{kg} & \mathrm{DM})\end{array}\right.\end{array}$ & 0,55 & 1,03 \\
\hline $\begin{array}{l}\text { Azote : MADC (g/kg MS) }{ }^{(2)} \ldots \ldots \ldots \ldots \ldots \\
\text { Nitrogen : } C D C P(g / k g \text { DM) }\end{array}$ & 48 & 124 \\
\hline
\end{tabular}

(1) Valeur énergétique : UFC (Unité Fourragère Cheval) - Energy value : HFU (Horse Feed Unit):

- du foin estimée à partir des relations établies entre la digestibilité de la matière organique et la composition chimique par MARTIN-Rosser et al. (1984) et des Nouvelles Tables INRA de la valeur nutritive des aliments du cheval (INRA, 1984) - of hay estimated from equations where organic matter digestibility and chemical composition of feedstuffs are related (MARTIN-RiossET and al., 1984) and from New Tables of nutritive value of feedstuffs for horses (INRA, 1984);

- de l'aliment concentré estimé à partir des équations INRA (1984) reliant la valeur énergétique et la composition chimique (MARTIN-ROSSET et al., 1984) - of concentrate estimated on the basis of INRA equations 1984 uhere energy value and chemical composition of feedstuffs are relased. (MARTIN-Rosser et al., 1984).

(2) Valeur azotée : MADC (Matières Azotées Digestibles Corrigées) - Crade protein content : CDCP (Corrected Digestible Crude Protein) :

- du foin et de l'aliment concentré estimée à partir des relations INRA établies entre la teneur en MADC et la teneur en MAT par MARTIN-Rosset et al. (1984) et des Nouvelles Tables de la valeur nutritive des aliments du cheval (INRA, 1984) - of hay and concentrate estimated from INRA equations where corrected digestible crude protein content is related to crude protein content of feedstuffs (MARTIN-ROSSET et al., 1984) and from New Tables of nutritive value of Feedstuffs for horses (INRA, 1984). 


\section{TABLeaU 2}

Apports alimentaires au cours de la période hivernale.

Dietary allowances during winter.

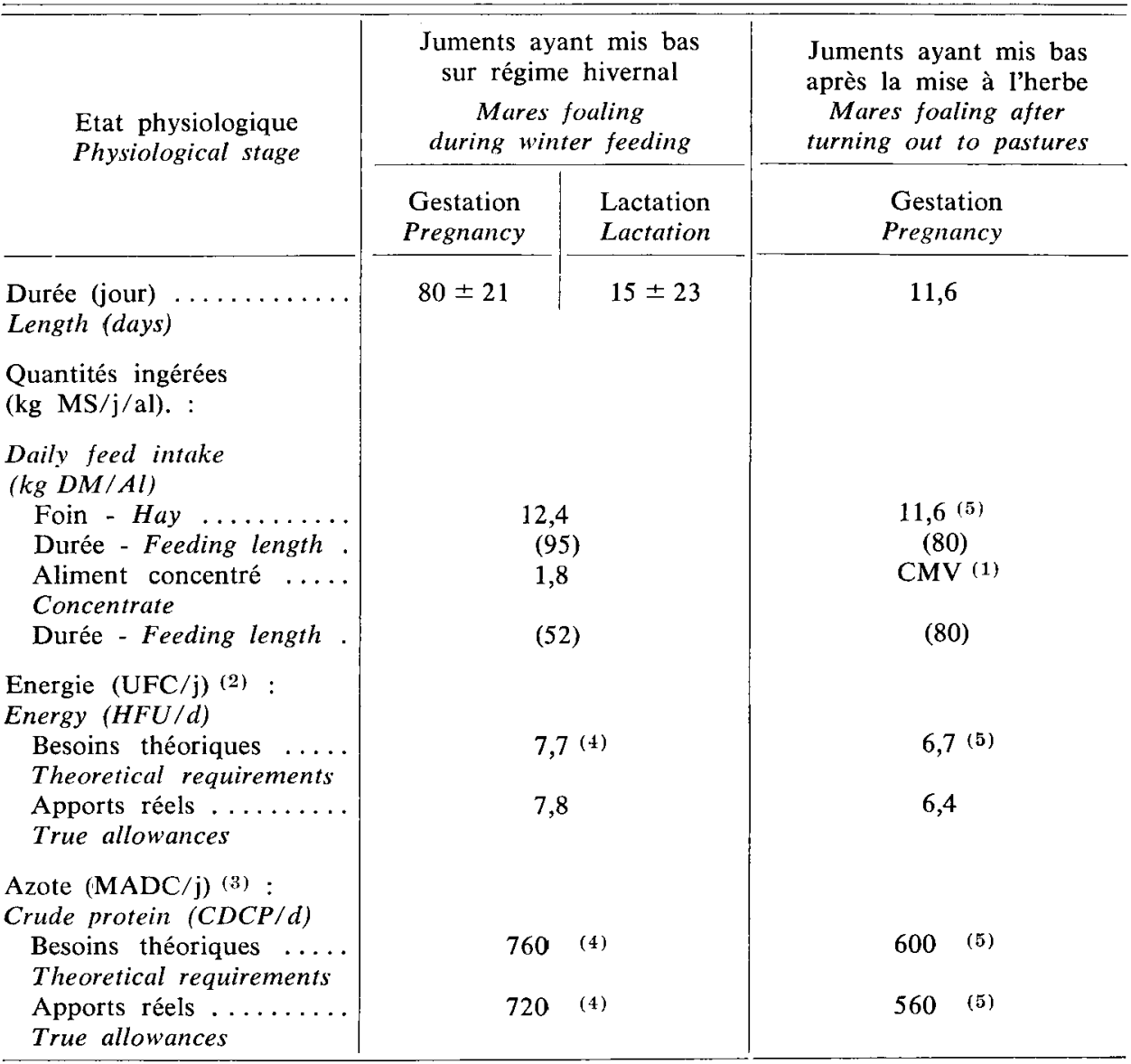

(1) CMV : Complément minéral et vitaminé : $100 \mathrm{~g} / \mathrm{j} / \mathrm{AI}$. MV : Mineral and vitamins : $100 \mathrm{~g} / \mathrm{d} / \mathrm{al}$.

(2) UFC : Unité Fourragère Cheval. HFU : Horse Feed Unit.

(3) MADC : Matières Azotées Digestibles Cheval. $C D C P$ : Corrected Digestible Crude Protein.

Système INRA 1984 - INRA-System 1984 VERMOREL - JARRIGE \& MARTIN-ROSSET, 1984 - JARRIGE \& TISSERAND, 1984

(4) Estimation à partir des besoins donnés dans les nouvelles tables des apports alimentaires recommandés pour le cheval, INRA 1984, pondérées par les durées moyennes de gestation et de lactation de la jument moyenne du troupeau. La production laitière des juments a été estimée à partir de la liaison établie par Doreau et al., 1986 entre la consommation de lait et la croissance du poulain - Evaluation from nutrient requirements given in the new tables of recommended dietary allowances for horses, INRA 1984, taking into account the average pregnancy and lactation length of a representative mare. Milk production of mares was evaluated from the relationship between milk intake and average daily gain of the foal (DOREAU et al., 1986).

(5) Evaluation à partir des besoins donnés dans les nouvelles tables des apports alimentaires recommandés pour le cheval, INRA 1984, pondérée par la durée de gestation de la jument moyenne du troupeau - Evaluation from nutrient requirements given in the new tables of recommended dietary allowances for Horses, INRA 1984, laking into account the average pregnancy length of a representative mare. 


\section{Quantités ingérées}

B. Mesures

Les quantités d'aliments distribuées ont été mesurées chaque jour globalement au niveau de chaque troupeau pour le foin et individuellement pour l'aliment concentré pendant toute la période hivernale. La quantité de foin distribuée a été ajustée au niveau du troupeau par rapport aux besoins physiologiques moyens du troupeau (i.e. pondérés par l'effectif de juments gestantes ou allaitantes de chaque troupeau, en tenant compte du stade physiologique de toutes les juments présentes). On a pu ainsi calculer les quantités de foin et d'aliment concentré consommées en moyenne par les juments, en fin de gestation et en début de lactation, sans distinguer toutefois les quantités ingérées correspondantes à chaque état physiologique puisque les juments gravides ou allaitantes sont conduites en lots pendant toute la période hivernale.

\section{Evolution du poids vif des juments et des poulains}

Les juments sont pesées chaque mois tout au long de l'année mais également au cours de la semaine précédant le poulinage. A la mise bas, la jument, son poulain, le placenta et l'amnios sont pesés. Ensuite, la jument et le poulain sont pesés tous les 8 jours pendant le $1^{\text {or }}$ mois puis tous les 15 jours pendant les $2^{\circ}$ et $3^{\circ}$ mois de lactation et seulement tous les mois ensuite. A chaque changement de régime les pesées ne sont effectuées que 15 jours après ce changement (régime hivernal) et 8 jours après mise à l'herbe.

\section{Poids vif des juments}

\section{Calculs}

Nous avons établi la courbe moyenne annuelle d'évolution du poids vif des juments adultes ayant pouliné 2 années consécutives en distinguant les femelles qui avaient mis bas pendant l'hiver (lot $\mathrm{A} ; \mathrm{n}=13$ ) ou après la mise à l'herbe (lot $\mathrm{B}$; $\mathrm{n}=6$ ) et allaité leurs poulains sans avoir eu de problèmes sanitaires.

Chaque point des courbes représente la moyenne des pesées individuelles des juments, à condition que pour un point donné de la courbe, les pesées individuelles correspondent à la fois à un stade physiologique aussi voisin que possible entre toutes les juments et à des pesées effectuées sur le même régime systématiquement à la même heure en début d'après-midi. Lorsqu'une pesée individuelle manquait on a interpolé entre les 2 pesées les plus proches effectuées sur un même régime dans les mêmes conditions et à des stades physiologiques comparables à celui des autres juments.

\section{Poids vif et gain de poids des poulains}

La courbe de poids des poulains $(n=35)$ a été établie par régression sur la base des nombreuses pesées individuelles effectuées (en moyenne par animal, $14 \pm 2$ ). L'équation ainsi obtenue a permis de calculer les poids des poulains à âge type. Les poids et gains de poids correspondant à des conditions de conduite, d'alimentation et de pâturage différentes : naissance - fin d'hiver - mise à l'herbe - montée estive - fin estive et sevrage, ont été calculés à partir des pesées réelles des animaux issus de juments multipares $(n=31)$. 
Les poids vits et les gains de poids moyens $(n=31)$ ont été comparés selon le sexe, le poids vif à la naissance à l'aide d'un test de $\mathrm{F}$ afin de vérifier l'homogénéité des variances, et d'un test de $t$ pour savoir si les moyennes sont significativement différentes ou non.

\section{Résultats et discussion}

\section{A. Evolution du poids vif des juments}

Les juments ont mis bas en moyenne à la mi-avril (lot A) ou à la mi-mai (lot B), soit 15 jours avant ou après la mise à l'herbe qui a eu lieu le 28 avril. Elles ont été alimentées selon leurs besoins nutritionnels pendant la période hivernale qui a couvert, pour les juments A, les 80 derniers jours de gestation et les 15 premiers jours de lactation, et pour les juments B, 80 jours au cours des $8^{\prime \prime}$, $9^{\circ}$ et $10^{\circ}$ mois de gestation (tabl. 2).

Le poids vif des juments augmente rapidement au cours des trois derniers mois de gestation (fig. 2 et 3 ). Le gain de poids vif, enregistré au cours de cette période, est pour les juments $A$ et $B$ respectivement de $51 \pm 20 \mathrm{~kg}$ et $65 \pm 22 \mathrm{~kg}$ pour des durées de gestation de $331 \pm 11$ jours et $340 \pm 12$ jours. L'augmentation n'est pas significativement différente entre les 2 lots. Dans les deux cas on observe un léger fléchissement du poids vif au cours des 2 dernières semaines de gestation. Le gain de poids vif représente respectivement 7,3 et 9,0 p. 100 du poids vif des juments 24 heures après mise-bas. Ces valeurs sont intermédiaires entre celles observées chez la jument de selle (6 à 7 p. 100) par OTt (1970), Dusek \& Richter (1972), et celles rapportées par JoRdAN (1979) chez la ponette (10 à 13 p. 100).

Le gain de poids vif observé au cours des 3 derniers mois de gestation correspond à l'accroissement du poids du conceptus (fœetus + placenta + amnios + liquides fœtaux + utérus). D'après le bilan nutritionnel rapporté au tableau 2, les juments n'ont pas dû mobiliser ou constituer de réserves corporelles. Doreau, Martin-Rosset \& BARLET (1981) n'ont pas observé, chez des juments conduites dans les mêmes conditions, de mobilisation des réserves corporelles, appréciées par la variation de la teneur sanguine en acides gras non estérifiés (AGNE), au cours de cette période, lorsque les juments sont alimentées selon leurs besoins. Le placenta, l'amnios, les liquides fœtaux et l'utérus se développent au cours de la gestation plus précocement que le fœtus (Douglas \& GinTHER, 1975). Ils représentent 60 à 65 p. 100 du poids du conceptus à la mi-gestation et seulement 35 à 40 p. 100 à la mise bas (ArTHUR, 1969 ; DOuglas \& Ginther, 1975). 60 à 77 p. 100 du poids du fœtus sont acquis au cours des 3 derniers mois de gestation (Dusek, 1966 ; Meyer \& Ahlswede, 1976 ; Platt, 1984) ; le gain de poids que nous avons enregistré au cours de la même période correspond donc essentiellement à un accroissement du poids du fotus et il représente 73 p. 100 de celui-ci à la naissance.

Au poulinage, les juments $\mathrm{A}$ et $\mathrm{B}$ perdent respectivement 99 et $88 \mathrm{~kg}$ soit environ 12 p. 100 de leur poids vif après mise bas, valeur comparable à celle observée par GoATER et al. (1981). Les poulains pèsent $69,5 \mathrm{~kg}$ en moyenne à la naissance $(n=13+6)$, ce qui représente 73 p. 100 de la perte de poids vif observée. Les annexes (placenta + amnios) pèsent en moyenne $6,1 \pm 1,3 \mathrm{~kg}$, soit $6,4 \mathrm{p} .100 \mathrm{de}$ 


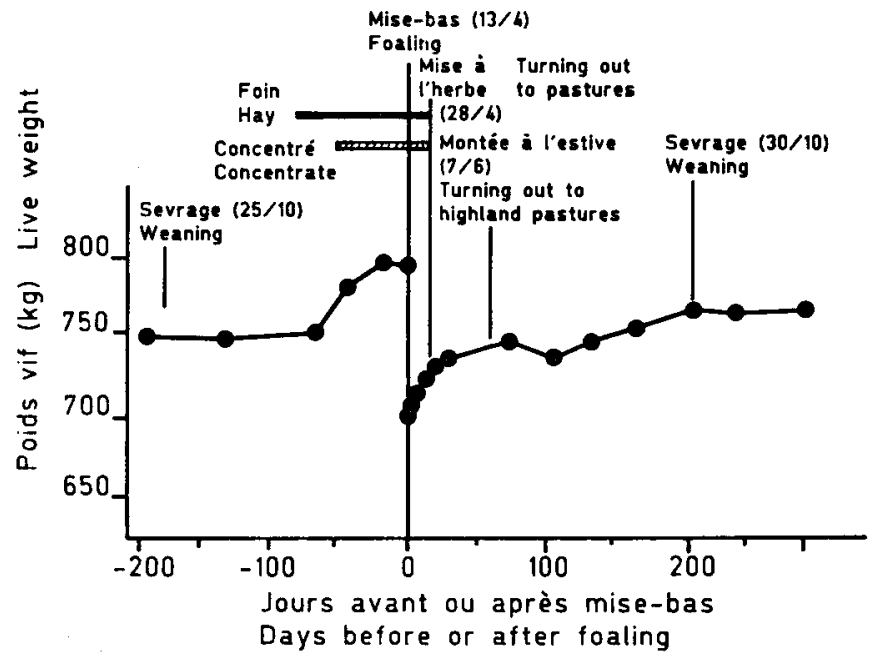

FIG. 2

Evolution du poids vif des juments ayant mis bas sur régime hivernal ( $n=13)$.

Variation in the live weight of mares foaling during the winter $(n=13)$.

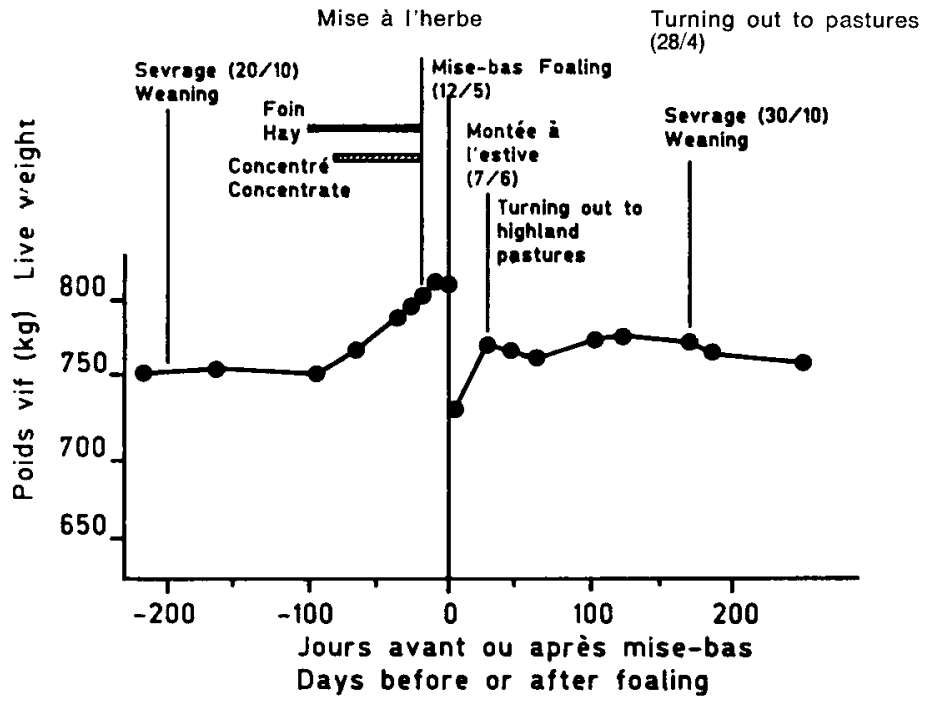

FIG. 3

Evolution du poids vif des juments ayant mis bas sur régime herbe $(n=6)$. Variation in the live weight of mares foaling during the grazing season $(n=6)$. 
la perte instantanée de poids de la jument à la mise bas. Si le poids du contenu digestif de la jument varie peu en fin de gestation et à la mise bas, les fluides fotaux représentent 20,8 p. 100 de la perte de poids soit $19,9 \mathrm{~kg}$, ce qui est en moyenne supérieur aux poids ou volumes rapportés par Richter \& Gotze (1960), Dickerson et al. (1967), Arthur (1969), chez la ponette ou la jument de selle. La variation du poids du contenu digestif à la mise bas peut expliquer sans doute la différence.

Après la mise bas et jusqu'à la montée en estive la jument gagne immédiatement du poids : en moyenne 40 à $45 \mathrm{~kg}$ (fig. 2 et 3 ). La reprise de poids est d'autant plus rapide que les juments sont au pâturage. Les quantités ingérées mesurées chez la jument lourde, dans le cadre d'autres essais (MARTin-Rosset \& DorEAU, 1984), s'accroît de $1,2-1,5 \mathrm{~kg} \mathrm{MS} / 100 \mathrm{~kg}$ de poids vif en fin de gestation ( $\left(9^{\circ}-11^{\circ}\right.$ mois) à $3,0 \mathrm{~kg} \mathrm{MS} / 100 \mathrm{~kg}$ de poids vif en début de lactation $\left(1^{\circ{ }^{\circ}-2^{\mathrm{e}}}\right.$ mois $)$ comme chez la jument de selle (N.R.C., 1978). Le contenu digestif représente 10 à 12 p. 100 du poids vif de la jument lourde à l'abattage soit 70 à $80 \mathrm{~kg}$ (MARTIN-Rosset, Doreau \& Robelin, 1986). Les juments du lot $\mathrm{A}$ ont perdu $50 \mathrm{~kg}$ entre les pesées du $9^{\mathrm{e}}$ mois de gestation et 24 heures après mise bas. Cette perte de poids correspond pour partie au moins à une variation du contenu digestif puisque les juments n'ont pas mobilisé de réserves corporelles, d'après le bilan énergétique rapporté au tableau 2, et que la teneur en AGNE reste constante au cours de cette période chez des animaux conduits dans les mêmes conditions (Doreau, Martin-Rosset \& Barlet, 1981). Toutefois on ne peut conclure avec plus de précision actuellement tant que l'on n'a pas d'une part mesuré individuellement chez la jument en fin de gestation et en début de lactation l'évolution des quantités ingérées d'une ration de composition constante, et d'autre part les variations concomitantes du poids du contenu digestif et des quantités ingérées.

Au cours de la période de pâturage estival, les juments n'ont gagné que $11 \mathrm{~kg}$ en moyenne (respectivement 22 et $2 \mathrm{~kg}$ pour les lots A et B). Ce résultat confirme indirectement que les juments des 2 lots ont été alimentées correctement en gestation pendant la période hivernale mais que les apports alimentaires en début de lactation ( $1^{\circ r}$ mois) ont été plus limitants pour le lot A conduit en parc d'hivernage, que pour le lot $\mathrm{B}$ au pâturage, compte tenu de la valeur nutritive respective du foin distribué et de l'herbe pâturée au printemps. Au tarissement après 180 jours de lactation, les poids vifs des juments des lots A et B sont comparables : respectivement 760 et $753 \mathrm{~kg}$, et égaux ou supérieurs à ceux observés à l'issue du sevrage de l'année précédente. Le gain de poids vif de la jument entre la mise bas et le sevrage est égal à 63 et $47 \mathrm{~kg}$ pour les lots $\mathrm{A}$ et $\mathrm{B}$. La jument mobilise au cours de l'automne une partie de ce gain, de 5 à $20 \mathrm{~kg}$, entre le tarissement et le début de la période d'alimentation hivernale suivante.

\section{B. Poids vif et croissance des poulains}

L'évolution du poids vif et du croît à âges types des poulains a été décrite pour tous les poulains nés de juments multipares et sevrés : soit $\mathrm{n}=31$ (fig. 4).

Le poids vif moyen des poulains à la naissance a été de $65,9 \pm 7,4 \mathrm{~kg}$, ce qui représente 9,8 p. 100 du poids vif de la mère le jour de la mise bas. Il est voisin de celui mesuré chez le cheval de trait belge par Olsson (1952) ou Olofsson \& THомке (1963). Au sevrage à $196 \pm 29$ jours, les poulains ont pesé $326,5 \pm 43,7 \mathrm{~kg}$. Le poids vif ( $\mathrm{Y}$ en $\mathrm{kg}$ ) est lié à l'âge ( $\mathrm{t}$ en jours) du poulain. 


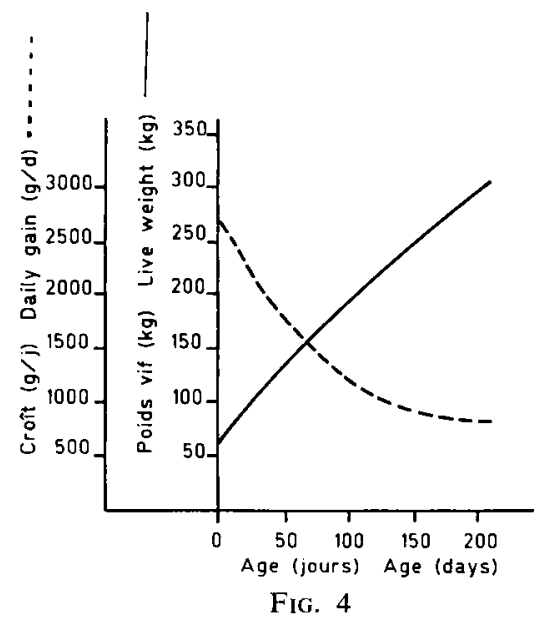

Evolution du crôt journalier et du poids vif des poulains $(n=31)$ (données régression). Variation in the daily gain and live weight of foals with age (regression data).

$$
\mathrm{Y}=87,49+1,25 \mathrm{t} \quad \mathrm{Syx}=21,6 \quad \mathrm{R}^{2}=0,939
$$
en $t^{3}$.

La relation est significativement améliorée après un ajustement curvilinéaire

$$
\begin{aligned}
& Y=68,57+2,1217 t-0,0066 t^{2}+0,00001 t^{3} \\
& S y x=18,6 \quad R^{2}=0,954
\end{aligned}
$$

Les poulains ont eu un croît moyen journalier de la naissance au sevrage de $1333 \pm 103 \mathrm{~g}$, très comparable à celui mesuré par OLsson (1952), OLOFsSON \& Thомке (1963), chez le cheval de trait belge, mais bien supérieur à celui observé chez le cheval de selle (Revue de MARTIN-Rosset, 1983). Le poulain double son poids de naissance à 30-35 jours (fig. 4) car son croît journalier est très élevé au cours de cette période : $2122 \mathrm{~g}$. Jusqu'à l'âge de 1 mois, le poulain ne consomme que le lait maternel (MARTIN-Rosset, DoREAU \& Cloix, 1978) et sa croissance est donc très liée à la production laitière de la jument (Oftedal et al., 1983 ; Doreau et al., 1986). Au-delà de 1 mois, la relation est plus lâche. 11 est vraisemblable que les différences individuelles dans l'utilisation des nutriments, la composition du gain de poids et le besoin d'entretien augmentent avec l'âge. La croissance du poulain dépend également de la quantité et de la qualité de l'herbe consommée. La part des besoins d'entretien dans les besoins totaux du poulain s'accroît avec l'âge. La composition du gain de poids vif évolue également avec l'âge : la part de l'eau dans le gain de poids vif diminue alors que la part des lipides augmente et corrélativement la valeur énergétique $\mathrm{du} \mathrm{kg}$ de gain aussi. C'est pourquoi au-delà de 1 mois la croissance du poulain diminue (fig. 4) : à deux mois le croît journalier est encore de $1300 \mathrm{~g}$ mais après 3 mois il n'est plus que de $1000 \mathrm{~g}$ et ne dépasse pas $900 \mathrm{~g}$ avant le sevrage.

La vitesse de croissance des poulains, mesurée entre la naissance et le sevrage, est liée significativement au poids à la naissance mais la liaison est faible $(r=0,51$; $\mathrm{P}<0,05 ; \mathrm{n}=31$ ). 
Le poids vif des mâles $(n=16)$ et des femelles $(n=15)$ à la naissance n'a pas été significativement différent : respectivement $65,6 \pm 8,8 \mathrm{~kg}$ et $66,2 \pm 5,8 \mathrm{~kg}$, tout comme les poids vifs au sevrage : $321,0 \pm 40,3 \mathrm{~kg}$ à $193 \pm 31 \mathrm{j}$ et $332,4 \pm 47,8 \mathrm{~kg}$ à $198 \pm 26 \mathrm{j}$.

Les poulains nés 15 jours avant $(\mathrm{n}=19)$ ou après $(\mathrm{n}=12)$ la mise à l'herbe n'ont pas un poids vif à la naissance significativement différent : respectivement $66,3 \pm 8,7 \mathrm{~kg}$ et $68,5 \pm 7,3 \mathrm{~kg}$ (fig. 5). Les poulains nés en hiver ont présenté au cours des mois suivant la naissance une croissance élevée, de 1800 à $1900 \mathrm{~g}$, et régulière aussi bien pendant l'hiver qu'au cours du pâturage au printemps. Ce croît est inférieur à celui observé au printemps au cours du premier mois suivant la naissance chez les poulains nés après la mise à l'herbe : plus de $2000 \mathrm{~g}$ (fig. 5). Mais comparés à même âge type, les poids des poulains nés avant ou après la mise à l'herbe ne sont pas significativement différents (tabl. 3). Plusieurs auteurs (SHEPETov, Mulikuv \& Gaidash, 1953 ; Doreau et al., non publié) ont montré qu'un accroissement des apports énergétiques chez la jument se traduit le plus souvent par une augmentation de sa production laitière et corrélativement de la croissance des poulains. Chez la vache allaitante, vêlant après la mise à l'herbe, le niveau de production laitière initiale est beaucoup plus élevé (de 20 à 25 p. 100) que celui des

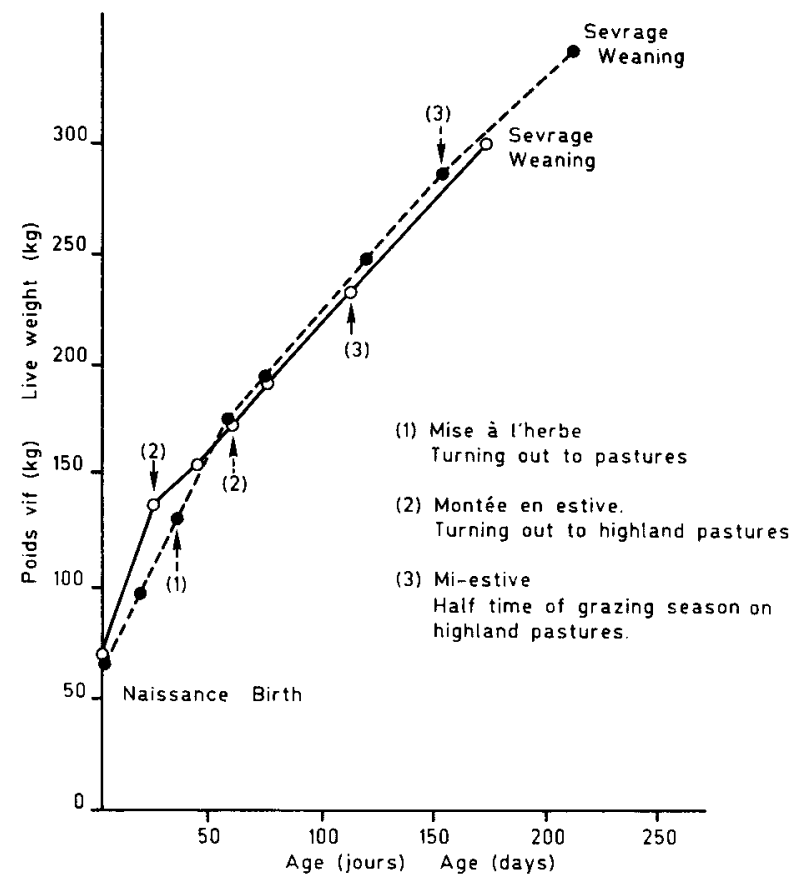

FIG. 5

Evolution comparée du poids vif des poulains nés avant (•) $n=19$ ou après (O) $n=12$ la mise à l'herbe (données réelles).

Compared variations in the live weight of foals born before (•) $n=19$ or after (O) $n=12$ turning out to pastures (real data). 


\section{TABLEAU 3}

Poids vifs et crô̂ts journaliers à âges types des poulains.

Live-weight and daily gain of foals at standard ages.

\begin{tabular}{|c|c|c|}
\hline & $\begin{array}{c}\text { Nés avant } \\
\text { la mise à l'herbe } \\
\text { Born before turning out } \\
\text { to pastures }(\mathrm{n}=19)\end{array}$ & $\begin{array}{l}\text { Nés après } \\
\text { la mise à l'herbe } \\
\text { Born after turning out } \\
\text { to pastures }(\mathrm{n}=12)\end{array}$ \\
\hline \multicolumn{3}{|l|}{ Poids vifs $(\mathrm{kg})$ : } \\
\hline Live-weight $(\mathrm{kg})$ & & \\
\hline Poids naissance - Birth weight ...... & $63,3 \pm 7,3$ & $69,9 \pm 5,7$ \\
\hline $30 \mathrm{j}-d \ldots \ldots \ldots \ldots \ldots \ldots \ldots \ldots$ & $126,3 \pm 12,2$ & $129,9 \pm 13,1$ \\
\hline $45 \mathrm{j}, \ldots \ldots \ldots \ldots \ldots \ldots \ldots \ldots \ldots \ldots \ldots$ & $152,5 \pm 14,7$ & $154,5 \pm 16,0$ \\
\hline $90 \mathrm{j}$ & $217,3 \pm 19,4$ & $213,1 \pm 19,7$ \\
\hline $180 \mathrm{j}$ & $310,6 \pm 25,9$ & $313,3: \pm 18,4$ \\
\hline \multicolumn{3}{|l|}{ Croîts journaliers $(\mathrm{g} / \mathrm{j})$} \\
\hline Daily gain $(g / d)$ & & \\
\hline $0-1$ mois - Months & $2098 \pm 286$ & $1999 \pm 349$ \\
\hline $0-3$ mois $\ldots \ldots \ldots \ldots$ & $1710 \pm 173$ & $1591 \pm 183$ \\
\hline $1-3$ mois $\ldots \ldots \ldots \ldots \ldots \ldots \ldots \ldots$ & $1516 \pm 140$ & $1388 \pm 137$ \\
\hline 3-6 mois $\ldots \ldots \ldots \ldots \ldots \ldots \ldots \ldots$ & $1036 \pm 167$ & $1117 * \pm 191$ \\
\hline $0-6$ mois $\ldots \ldots \ldots$ & $1373 \pm 117$ & $1341^{*} \pm 97$ \\
\hline
\end{tabular}

$*(n=6)$

vaches vêlant au cours de l'hiver (LE NEINDRE, 1974). Les juments qui ont pouliné après la mise à l'herbe ont reçu une alimentation plus libérale que celles qui ont mis bas pendant l'hiver. Or il semble d'après Doreau, Martin-Rosset \& Barlet (1981) qu'à partir d'une certaine consommation d'énergie tout apport supplémentaire se partage entre la production laitière et le gain de poids de la jument jusqu'à la réalisation du potentiel laitier.

Le pâturage d'été n'a pas permis aux poulains nés tôt d'avoir une croissance $(1120 \pm 138 \mathrm{~g} / \mathrm{j})$ très différente de celle des poulains nés tardivement $(1043 \pm$ $168 \mathrm{~g} / \mathrm{j}$ ). Dans ces conditions on n'a pas observé de différence significative du croît entre la naissance et le sevrage pour les poulains nés pendant l'hiver ou au pâturage : respectivement, $1308 \pm 103 \mathrm{~g}$ et $1350 \pm 95 \mathrm{~g}$. Mais les poids au sevrage ont été significativement plus élevés chez les poulains nés tôt : $342,2 \pm 39,0 \mathrm{~kg}$ que chez les poulains nés tard : $299,3 \pm 37,1 \mathrm{~kg}$, compte tenu de la différence d'âge au sevrage, soit 40 jours. 


\section{Conclusion}

Le poids vif de la jument est un critère utile mais relativement global pour évaluer avec précision, dans le cadre d'essai d'alimentation, l'influence du niveau des apports alimentaires au cours du cycle Gestation-Lactation. Il est le résultat de la variation simultanée de trois composantes : le conceptus en fin de gestation, le contenu digestif et les réserves corporelles. On ne peut facilement les dissocier pour interpréter le fléchissement du poids vif de la jument au cours des 2 dernières semaines de gestation ou au contraire lors de l'accroissement important de son poids en début de lactation, si on ne dispose pas au préalable d'une estimation des variations du poids du contenu digestif rapportées à celles des quantités ingérées mesurées individuellement, et d'une méthode d'estimation indirecte de l'état des réserves corporelles.

Le poulain présente une croissance élevée, en particulier au cours du premier mois de lactation. Celle-ci est étroitement liée à la production laitière de la mère $(\mathbf{r}=0,8)$, la quantité de lait consommée par $\mathrm{kg}$ de gain de poids étant de $10 \mathrm{~kg}$ (Bouwman \& Van der Schee, 1978 ; Doreau, Martin-Rosset \& Dubroeuco, 1982 ; Oftedal, Hintz \& Schryver, 1983 ; Doreau et al., 1985). Néanmoins, au-delà de l'âge de 1 mois, la liaison est plus lâche $(r<0,8)$ car la croissance du poulain dépend également de la quantité et de la qualité de l'herbe pâturée (MARTIN-Rosset, DoREAU \& Cloix, 1978 ; Duncan, 1980). Le coût énergétique de production du $\mathrm{kg}$ de gain augmente avec l'âge : il passe de 5,8 à 6,5 Mcal entre le $1^{\text {er }}$ et le $2^{\text {c }}$ mois (Doreau et al., 1986) et la variabilité individuelle s'accentue. La croissance du poulain se révèle donc être un bon critère d'estimation de la production laitière de la jument au cours du $1^{\text {er }}$ mois, quoiqu'elle permette seulement d'estimer les variations de cette production, dans l'état actuel des connaissances.

Reçu en juillet 1985.

Accepté en décembre 1985.

\section{Summary}

Suckling mare feeding : variation in the mare live weight and foal growth

Variation in the live weight of well fed heavy brood mare (tables 1 and 2) during pregnancy and lactation cycle, and growth of the suckled foal have been studied in 35 couples.

During the last three months of pregnancy the live weight of mares increased by 8 p. 100 (fig. 2 and 3). This weight gain corresponded to the conceptus development, mainly the foetus, and represented 73 p. 100 of the foal live weight at birth.

One month after foaling live weight of the suckling mare increased by 6 to 7 p. 100 (fig. 2 and 3). Variation in the digestive content might partly account for the weight loss observed two weeks before foaling and for the weight increase in early lactation. We did not notice any significant difference according as to whether foaling occurred before turning out to pasture or after.

The foal live weight at birth represented $10 \mathrm{p} .100$ of that of the mare at foaling and was closely related with its own age. Average daily gain of the foal from birth to weaning 
was $1300 \mathrm{~g}$ : it was very high during the first month at the end of which the foal live weight was twice higher than that at birth, for it was closely related with the mare milk yield (fig. 4). There was no significant difference between sex or date of birth relative to turning out to pasture (fig. 5 and table 3 ).

The mare live weight was a useful criterium but not accurate enough to study the effect of feeding during pregnancy and lactation.

On the other hand the foal growth was a good criterium for estimating the mare milk yield and its variation with the level of feeding during the first month of lactation.

Key words : horse, feeding, mare, foal, live weight.

\section{Références bibliographiques}

ANashina N.V., Koveshnikov V.S., 1982. Economic and organizational basis for commercial milk and meat production in horse breeding. 33rd Annual meeting EAAP, Leningrad, 16-19 août. Horse Commission, Session 6, 1-5 (en russe).

Archambeaud B., Jussiaux M., Palmer E., Signoret J.P., 1973. Méthode de détection de l'œstrus chez la jument en liberté. Ann. Zootech., 22, 3, 33-95.

Arthur G.H., 1969. The fetal fluids of domestic animals. J. Repr. Fert., Suppl. 9, 45-52.

BARMINSTEV Y.N., 1982. Genetic anr organizational basis for the increase of milk and meat production in Horse breeding, 33rd Annual meeting EAAP, Leningrad, 16-19 août. Horse Commission, Session 6, 1-10 (en russe).

Bouwman H., VAN DeR ScheE W., 1978. Composition and production of milk from Dutch Warm blooded saddle horse mares. Z. Tierphysiol. Tierernährg. Futtermittelk, 40, 39-53.

Butaye R., 1966. Poids et croissance de poulains et de chevaux plus âgés chez la race de trait belge. Klaams Diergeneeskundig. Tijdschsift., 35, 157-175 (en flamand).

Dickerson J.W.T., Soutghate D.T., King J.M., 1967. The origin and development of the hippomanes in the horse and zebra. 2. - The chemical composition of the fetal fluids and hippomanes. J. Anat., 101, 285.

Doreau M., Martin-Rosset W., Barlet J.P., 1981. Variations de quelques constituants plasmatiques chez la jument allaitante en fin de gestation et en début de lactation. Ann. Rech. Vet., 12, 219-225.

Doreau M., Martin-Rosset W., Dubroeuce H., 1982. Production laitière de la jument. Liaison avec la croissance du poulain. 8* Journée d'étude. Cereopa Fd., 16, rue ClaudeBernard, 75231 Paris Cedex 05, 88-100.

Doreau M., Boulot S., Martin-Rosset W., Robelin J., 1986. Relatonship between nutrient intake, growth and body composition of the nursing foal. Reprod. Nutr. Develop., 26 (sous presse).

Douglas R.H., Ginter O.J., 1975. Development of the equine fetus and placenta. J. Repr. Fert., Suppl., 23, 503-505.

Duncan P., 1980. Time budgets of Camargue horses. II. - Time budgets of adult horses and weaned sub-adults. Behaviour, 72, 26-49.

DusEK J., 1966. Influence de la durée de gestation sur les performances ultérieures des poulains. Vedecké Vysk. Bull., 2, 49-65 (en tchèque).

Dusek J., Richter L., 1972. Anderungen der korpermasse von Stuten im Verlaufe der Gravidität. Arch. Tierzucht., 15, 361-366.

Goater L.E., Meacham T.N., Gwazdauskas F.C., Fontenot J.P., 1981. The effect of feeding excess energy to mares during late gestation. Proc. 7th Eq. Nutr. Physiol. Symp., Warrenton, Virginie, U.S.A., 111-115.

Groupe de Recherches sur les haUts paturages dégradés des Monts Dore, 1979. Aspects biologiques et techniques de la remise en exploitation des hauts pâturages dégradés des Monts Dore. In : Utilisation par les ruminants des pâturages d'altitude et parcours méditerranéens. INRA Publications, route de Saint-Cyr, 78000 Versailles, p. 57-155. 
Henneke D.R., Potter G.D., KreIDer J.L., 1981. Rebreeding efficiency in mares fed different levels of energy during late gestation. Proc. 7th Eq. Nutr. Physiol. Symp., Warrenton, Virginie, U.S.A., 101-104.

INRA, 1984. Tables des apports alimentaires recommandés pour le cheval. $I n:$ R. JaRRIGE \& W. Martin-Rosset, Ed., «Le Cheval. Reproduction - Sélection - Alimentation Exploitation». INRA Publications, route de Saint-Cyr, 78000 Versailles, p. 465-469.

INRA, 1984. Tables de la valeur nutritive des aliments pour le cheval. In : R. JARRigE \& W. Martin-Rosset, Ed., «Le Cheval. Reproduction - Sélection - Alimentation Exploitation». INRA Publications, route de Saint-Cyr, 78000 Versailles, p. 661-689.

ISAEVA O.S., 1982. Milk performance of mares belonging to the Russian and Soviet draft breeds. 33rd Annual meeting EAAP, Leningrad, 16-19 août. Horsc Commission, Session 6, 1-4 (en russe).

Jarrige R., Tisserand J.L., 1984. Métabolisme, besoins et alimentation azotés du cheval. In : R. Jarrige \& W. Martin-Rosset, Ed., "Le Cheval. Reproduction - Sélection Alimentation - Exploitation». INRA Publications, route de Saint-Cyr, 78000 Versailles, p. $276-302$.

JoRdan R.M., 1979. A note on the energy requirements for lactating pony mares. Proc. 6th Eq. Nutr. Physiol. Symp., Texas, U.S.A., 27-32.

LE NeIndRe P., 1974. Production laitière des vaches allaitantes et liaison entre cette production et la croissance de leurs veaux. In : R. JARRIGE, Exploitation des troupeaux de vaches allaitantes. $\mathrm{N}^{*}$ spécial Bull. Tech. INRA, C.R.Z.V. Theix, p. 212-230.

Martin-Rosset W., Doreau M., Cloix J., 1978. Etude des activités d'un troupeau de poulinières de trait et de leurs poulains au pâturage. Ann. Zootech., 27, 33-45.

Martin-Rosset W., Doreau M., 1980. Effect of variation in level of jeeding of heavy mares during late pregnancy. Proc. 31st Annual Meeting EAAP. Munich. Horse Commission, Session 6, 1-4.

Martin-Rosset W., Loiseau P., Molenat G., 1981. Utilisation des pâturages pauvres par le cheval. B.T.I., 362-363, 587-608.

Martin-Rosset W., 1983. Particularités de la croissance et du développement du cheval. Ann. Zootech., 32, 109-130.

Martin-Rosset W., Trillaud-Geyl C., 1984. Exploitation des troupeaux de juments lourdes allaitantes. In : R. JARrige \& W. MARTIN-Rosset, "Le Cheval. Reproduction - Sélection - Alimentation - Exploitation. INRA, Paris, Publication, route de Saint-Cyr, 78000 Versailles, p. 541-554.

Martin-Rosset W., Doreau M., 1984. Besoins et alimentation de la jument. In : R. JARrige \& W. Martin-Rosset, Ed., «Le Cheval. Reproduction - Sélection - Alimentation - Exploitation». INRA Publications, route de Saint-Cyr, 78000 Versailles, p. $355-370$.

Martin-Rosset W., Andrieu J., Vermorel M., Dulphy J.P., 1984. Valeur nutritive des aliments pour le cheval. In : R. JARrige \& W. MarTIN-RosseT, Ed., «Le Cheval. Reproduction - Sélection - Alimentation - Exploitation». INRA Publications, route de Saint-Cyr, 78000 Versailles, p. 208-238.

Martin-Rosset W., Doreau M., Robelin J., 1986. Estimation in vivo des tissus adipeux chez la jument par la méthode de dilution de l'eau lourde ou par la taille des adipocytes. (En préparation).

Meyer H., Ahlswede L., 1976. Uber das intrauterine Wachstum und die Körperzusammensetzung von Stuten in verlaufe der Laktation. Ubers. Tierernährg., 4, 263-292 (en allemand).

N.R.C., National Research Council, 1978. Nutrient requirements of horses. $\mathrm{N}^{\circ}$ 6. 4th revised ed. National Academy of Science Ed., Washington D.C., p. 33.

Oftedal O.T., Hintz H.F., Schryver H.F., 1983. Lactation in the horse : milk composition and intake by foals. J. Nutr., 113, 2196-2206.

Olofsson N.E., Thomke S., 1963. Raising experiments with young horses at Ultuna, 19521958. Lantbrukshogskolaus meddelanden, série A, n" 1, Uppsala, 3-22.

OLsson N.O., 1952. La consommation d'aliment et la croissance des chevaux Ardennais. Kungl. Lantbrukshogskdan och st. Lantbruksforsok, 49, 1-13 (en suédois). 
OTT E.A., 1970. Energy and protein for reproduction in the horse. Proc. 2nd equine nutrition research symposium. Ithaca, New York, 6-10.

Pagan J.D., Hintz H.F., 1981. Digestible energy requirements of lactating pony mares. Proc. 7th Equine Nut. Physiol. Symp., Warrenton, Virginie, U.S.A., 121-126.

PlatT M., 1984. Growth of the equine foetus. Eq. Vet. J., 16, 247-252.

Richter J., Gotze T., 1960. Tiergeburtshilfe, Paul Parey, Berlin et Hambourg. Cité par ARTHUR, 1969.

Rossier E., 1984. Etat actuel de la production et de la consommation de viande chevaline en France. In : R. JARrige \& W. Martin-Rosset, «Le Cheval. Reproduction Sélection - Alimentation - Exploitation». INRA, Publications, Route de Saint-Cyr, 78000 Versailles, p. 491-508.

Shepetov F.N., Mulikov A.I., GaIdash V.A., 1953. L'élevage des chevaux pour le lait dans la région de Stalingrad. Konevod. Konnyj sport, 6, $41-45$ (en russe).

Sutton E.I., Bowland D.P., Ratcliff W.D., 1977. Influence of level of energy and nutrient intake by mares on reproductive performance and on blood serum composition of the mares and foals. Can. J. Anim. Sci., 57, 551-558.

Vermorel M., JARrige R., Martin-Rosset W., 1984. Métabolisme et besoins énergétiques du cheval. Le système des UFC. In : R. Jarrige \& W. Martin-Rosset, Ed., «Le Cheval. Reproduction - Sélection - Alimentation - Exploitation». INRA Publications, route de Sainy-Cyr, 78000 Versailles, p. 238-276.

Zimmerman R.A., 1981. Energy needs of lactating mares. Proc. 7th Equine Nutr. Physiol. Symp., Warrenton, Virginie, U.S.A., 127-135. 